\title{
Age at menarche is associated with prediabetes and diabetes in women (aged 32-81 years) from the general population: the KORA F4 Study
}

\author{
D. Stöckl • A. Döring • A. Peters • B. Thorand • \\ M. Heier • C. Huth • H. Stöckl • W. Rathmann • \\ B. Kowall • C. Meisinger
}

Received: 16 July 2011 / Accepted: 21 November 2011 / Published online: 15 December 2011

(C) Springer-Verlag 2011

\begin{abstract}
Aims/hypothesis The aim of this study was to examine the
association between age at menarche and prediabetes as well
as diabetes, considering confounding factors and the possi-
ble mediating role of adult obesity.
Abstract
Aims/hypothesis The aim of this study was to examine the
association between age at menarche and prediabetes as well
as diabetes, considering confounding factors and the possi-
ble mediating role of adult obesity.

Abstract
Aims/hypothesis The aim of this study was to examine the
association between age at menarche and prediabetes as well
as diabetes, considering confounding factors and the possi-
ble mediating role of adult obesity.

Abstract
Aims/hypothesis The aim of this study was to examine the
association between age at menarche and prediabetes as well
as diabetes, considering confounding factors and the possi-
ble mediating role of adult obesity.

Abstract
Aims/hypothesis The aim of this study was to examine the
association between age at menarche and prediabetes as well
as diabetes, considering confounding factors and the possi-
ble mediating role of adult obesity.
\end{abstract}

D. Stöckl $(\bowtie) \cdot$ A. Peters $\cdot$ B. Thorand $\cdot$ M. Heier $\cdot$ C. Huth $\cdot$

C. Meisinger

Institute of Epidemiology II, Helmholtz Zentrum München,

German Research Center for Environmental Health,

Ingolstädter Landstr. 1,

D-85764 Neuherberg, Germany

e-mail: doris.stoeckl@helmholtz-muenchen.de

D. Stöckl

Department of Obstetrics and Gynaecology, Campus Grosshadern, Ludwig-Maximilians-University,

Munich, Germany

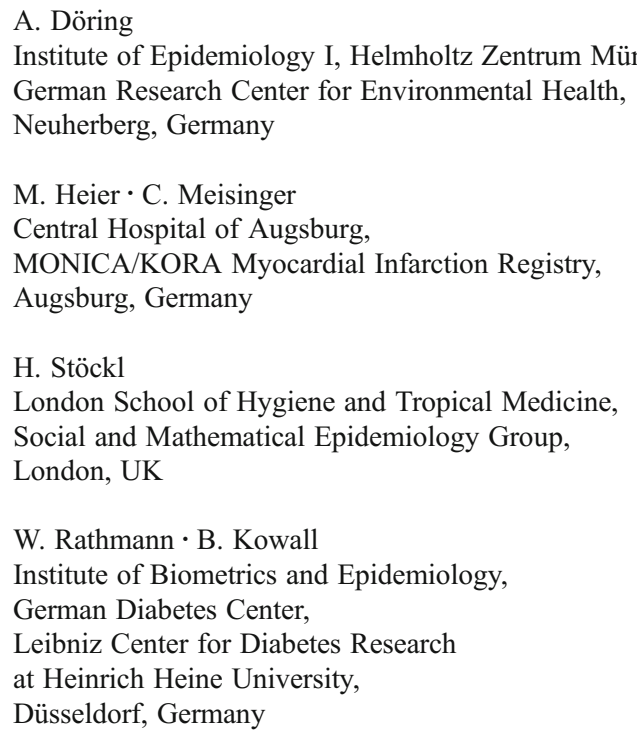

Düsseldorf, Germany

Methods This cross-sectional study analysed data on 1,503 women aged 32-81 years from the German populationbased KORA (Cooperative Health Research in the Region of Augsburg, South Germany) F4 Study (2006-2008). Data were collected by standardised interviews, physical examinations, and whole blood and serum measurements, including administration of an OGTT in non-diabetic participants. Results Of the 1,503 women, 226 showed a prediabetic state (impaired fasting glucose and/or impaired glucose tolerance) and 140 persons had diabetes (45 participants with previously undiagnosed diabetes and 95 with known diabetes). In Poisson regression analysis, age at menarche was significantly inversely associated with prediabetes or diabetes after adjustment for year of birth (RR 0.88; 95\% CI 0.82, $0.94, p<0.0001$ per additional year of menarche) and after additional adjustment for a number of confounding factors (RR 0.88; 95\% CI 0.83, 0.94, $p=0.0001$ ). Further adjustment for current BMI slightly attenuated the association with prediabetes or diabetes (RR $0.89 ; 95 \%$ CI $0.83,0.95$, $p=0.0009)$, but the association remained clearly significant. Conclusions/interpretation Age at menarche seems to be inversely associated with prediabetes and diabetes independent of confounding factors including current BMI. Women at risk for diabetes might be identified by a history of young age at menarche.

Keywords Diabetes · Impaired fasting glucose - Impaired glucose tolerance $\cdot$ Menarche $\cdot$ Menstruation $\cdot$ Prediabetes . Reproductive factors
Abbreviations
FG Fasting glucose
IFG Impaired fasting glucose
i-IFG Isolated impaired fasting glucose 
IGT Impaired glucose tolerance

i-IGT Isolated impaired glucose tolerance

KORA Cooperative Health Research in the Region of Augsburg, South Germany

NDD Newly diagnosed diabetes

NGT Normal glucose tolerance

SES Socioeconomic status

TG Triacylglycerol

WC Waist circumference

\section{Introduction}

Diabetes poses a significant health and economic burden for Westernised societies, which places high importance on the identification of preventive strategies and measures [1]. By the time type 2 diabetes mellitus is diagnosed, serious complications may have already developed [1]. For this reason it is important to identify persons at risk as early as possible to initiate preventive measures or treatment. Investigators of the KORA (Cooperative Health Research in the Region of Augsburg, F4 survey in Augsburg, Germany) study demonstrated that up to $50 \%$ of diabetes cases were undetected in the age group 55-74 years [2], and that there was already a high prevalence of impaired glucose regulation (isolated impaired fasting glucose [i-IFG], isolated impaired glucose tolerance [i-IGT] and combined IFG-IGT) in 35-59-yearold individuals [3].

Age at menarche was shown to be associated with higher BMI [4-7], cardiovascular disease [8-10], metabolic risk factors [4], the metabolic syndrome [11-13] and mortality $[9,11,12]$. Only a few studies have focused on the complex relationship between age at menarche and diabetes mellitus, with different results [5-7, 14]. In some studies the association between menarche and diabetes was discussed to be only caused by obesity [4], therefore the authors found it necessary to assess this topic again. The timing of menarche is mainly due to genetic factors, but is influenced by environmental factors [15]. The only study that examined association of age at menarche with IGT and type 2 diabetes did not find a significant association [7]. IGT is a known risk factor for type 2 diabetes and coronary heart disease [16]. Obesity, which is a major predictor of IGT and type 2 diabetes, is known to be associated with earlier age at menarche [17-19]. Whether or not there is an independent association of age at menarche with diabetes, or if there is only the link over obesity, needs to be assessed.

Therefore, the aim of this study was to determine whether there is an independent association between age at menarche and prediabetes (combination of i-IFG, i-IGT and IFG-IGT) as well as diabetes (newly diagnosed and known diabetes combined), or if this association is mediated only by BMI. Data from the population-based KORA F4 Study were used.

\section{Methods}

Subjects The KORA F4 (2006-2008) Study comprises 7 year follow-up data of the KORA S4 (1999-2001) Study, a population-based health survey conducted in the city of Augsburg and two surrounding counties. For the S4 baseline study, a total sample of 6,640 participants was drawn from the target population consisting of all German residents in the region aged 25-74 years. The study design, sampling method and data collection have been described in detail elsewhere [20,21].

Of all 4,261 S4 participants, 3,080 also participated in the F4 follow-up study. Individuals were not invited for F4 if they had died in the meantime $(n=176,4 \%)$, lived outside the study region or were lost to follow-up $(n=206,5 \%)$, or had demanded deletion of their address data $(n=12,0.2 \%)$. Of the remaining 3,867 eligible persons, 174 could not be contacted, 218 were unable to participate in the study because they were too ill or had no time, and 395 were not willing to participate in this follow-up, resulting in a response rate of $79.6 \%$. The current study was restricted to 1,594 female individuals, aged 32-81 years at follow-up.

All women for whom no, or incomplete, information was available on age at menarche $(n=33)$, or any of the covariables $(n=9)$, were excluded. Participants were classified as 'unknown glucose status' and excluded from the analysis if they had supplied inconsistent information about being diabetic or if their OGTT could not be completed correctly, because of nausea, no previous fasting period, etc. $(n=47)$. Furthermore, we excluded two women with age at menarche above 18, as described below. Therefore, the final analysis comprised 1,503 study participants.

The investigations were carried out in accordance with the Declaration of Helsinki, including written informed consent of all study participants. The study was approved by the Ethics Committee of the Bavarian Medical Association.

Data collection Trained medical staff obtained information on sociodemographic variables, physical activity level, medication use, alcohol consumption, smoking habits and reproductive history during a standardised face-to-face interview. Furthermore, all study participants underwent a standardised medical examination. All measurement procedures have been described in detail elsewhere [2, 20, 21]. Anthropometric measurements were taken after the participants had removed their shoes, heavy clothing and belts. Body height was measured to the nearest $0.1 \mathrm{~cm}$ and weight to the nearest $0.1 \mathrm{~kg}$. BMI was calculated as weight (in kilograms) divided by height ${ }^{2}$ (in square metres). Waist circumference (WC) was measured at the level midway between the lower rib margin and the iliac crest. BMI at age 25 years was assessed by recalled weight at age 25 , calculated as weight (in kilograms) divided by current 
height ${ }^{2}$ (in square metres). Actual hypertension was defined as blood pressure values greater than $140 / 90 \mathrm{mmHg}$ or the use of antihypertensive medication. Participants were classified as active during leisure time if they regularly participated in sports in the summer and winter and if they were active for $>1 \mathrm{~h} /$ week in either season. Women who consumed more than $20 \mathrm{~g}$ alcohol per day were regarded as heavy alcohol drinkers.

Clinical chemical measurements A fasting venous blood sample was obtained from all study participants while sitting. $\mathrm{HbA}_{1 \mathrm{c}}$ was measured using a reverse-phase cation-exchange HPLC method (analyzer HA 8160; Menarini, Florence, Italy). Serum glucose was analysed using a hexokinase method (GLU Flex), total cholesterol with a CHOD-PAP method (CHOL Flex), HDL cholesterol (HDL-C) with AHDL Flex and fasting triacylglycerol (TG) with the TGL Flex GPO-PAP assay (all assays from Dade Behring, Eschborn, Germany). These analyses and all additional were carried out using the analyzer Dimension RxL (Dade Behring).

Assessment of age at menarche The age at first menstrual bleeding assessed in full years was defined as age at menarche. This information was gained from the personal interview of the KORA S4 survey. The question was open-ended: 'At what age did you have your first menstrual period (menarche)?' We excluded participants with menarche later than 18 years $(n=2)$, because this is likely to be due to a pathological status or a recall error. For descriptive analysis, age at menarche was categorised into three categories $(<12,12-15$ and $>15$ years $)$ or used as continuous variable.

Assessment of glucose impairment and diabetes mellitus Known diabetes mellitus was defined as use of glucose-lowering medication or self-report, which was validated by questioning the treating physician. After an overnight fast of at least $10 \mathrm{~h}$, all non-diabetic participants underwent a standard $75 \mathrm{~g}$ OGTT. Blood was collected without stasis, refrigerated to $4-8^{\circ} \mathrm{C}$ and shipped on refrigerant packaging within 4 to a maximum of $6 \mathrm{~h}$ to the laboratory. Newly diagnosed diabetes (NDD), IFG, IGT and normal glucose tolerance (NGT) were defined according to the 1999 WHO diagnostic criteria based on both fasting and post-challenge glucose values [22]. We used the original IFG criteria $(6.1-6.9 \mathrm{mmol} / \mathrm{l})$ for the present analysis, as recommended by the European Diabetes Epidemiology Group [23].

Statistical analyses Basic characteristics of the study population were analysed stratified by the glucose status groups and stratified by age at menarche $(<12,12-15$ and $>15$ years $)$. For normally distributed variables the mean and standard deviation are presented, for non-normally distributed variables the median and the interquartile range are presented, and for categorical variables percentages are presented. $p$ values are shown for the difference of these variables between the three categories of age at menarche.

Fasting TG, HDL-C, fasting glucose (FG) and glucose $2 \mathrm{~h}$ post challenge during the OGTT (2-h glucose) were not normally distributed. Thus, the log-transformed normally distributed variables were used in all analyses. The results are presented as geometric means after back-transformation.

Three outcome variables were fitted: the three prediabetic states (i-IFG, i-IGT and IFG-IGT) were combined to one group called 'prediabetes' and the previously known and newly diagnosed diabetes groups to one group called 'diabetes'. As a further outcome variable prediabetes and diabetes were combined. Adjusted RR of diabetes (or prediabetes) onset were estimated by a generalised estimation equation approach including the natural logarithm of person-years of observation as offset term in the model. Years of observation were calculated from birth to the examination (age at examination) or to the year of onset of diabetes (age of onset of diabetes). The error term distribution was specified as Poisson with the usual natural log-link for a Poisson regression model [24]. We excluded diabetic women in the model for prediabetes. Prediabetic women in the model for diabetes were treated equally to women in the NGT group. Four models were fitted for each of the three outcomes. In the first model we controlled for year of birth. The second model controlled for the following confounding variables: year of birth, current smoking (yes/no), alcohol intake $>20 \mathrm{~g} /$ day (yes/no), physical activity regularly more than $1 \mathrm{~h}$ per week (yes/no), education $\leq 10$ years (yes/no), family status (married or living with a partner/living alone) and menopausal status (pre-/postmenopausal). The third model additionally controlled for current BMI (in kilograms per square metre). In a fourth model, current BMI was replaced with BMI at age 25 years (in kilograms per square metre).

A generalised linear model was used to examine the association between age at menarche and FG and 2-h glucose after performance of an OGTT. Stepwise confounder control was performed with the same confounders in the four models as described for the Poisson regression analyses.

Significance tests were two-tailed and $p$ values $<0.05$ were considered statistically significant. All analyses were performed using SAS (version 9.2, SAS Institute, Cary, NC, USA).

\section{Results}

Of the 1,503 women included in this analysis, 366 women had prediabetes $(n=226)$ or diabetes $(n=140)$ and were therefore classified into one of the five impaired glucose 
regulation and diabetes categories as described above (24.4\%). Age at menarche showed a normal distribution with a mean of 13.5 years (median 13 years), and a standard deviation of 1.6 years; the earliest reported age at menarche was 9 years.

Demographic and behavioural characteristics and risk factors stratified by glucose categories are shown in Table 1 . Women with prediabetes or diabetes were significantly older than non-diabetic women and were significantly more often postmenopausal. Diabetic women were more likely to have actual hypertension and less likely to be physically active. They also had a higher current BMI, a higher BMI at age 25 years and were less likely to be a current smoker. Women with diabetes had a higher percentage of being a widow, having had less education and were more likely to be multiparae.

Table 2 shows the basic characteristics by age at menarche categorised into three groups. There was a significant association between age at menarche and age, BMI at age 25 , menopausal status and physical activity.

The RRs for the associations between age at menarche (continuous) and prediabetes (combined i-IFG, i-IGT, and IFG-IGT), diabetes (newly and previously diagnosed diabetes combined) and combined prediabetes or diabetes are given in Table 3. Age at menarche was inversely associated with prediabetes and diabetes, after adjustment for year of birth. This result was still significant for both outcomes after additional adjustment for education, smoking status, alcohol intake, physical activity, marital status, year of prediabetes/ diabetes diagnosis and menopause status. Further adjustment for current BMI attenuated the association between age at menarche and both outcomes slightly, but the relationship remained clearly significant. After adjustment for BMI at age 25 instead of current BMI using the same calculations as in Table 3, the association between age at menarche and prediabetes and diabetes were quiet similar (RR $0.89 ; 95 \%$ CI $0.83,0.95$ for prediabetes or diabetes combined, RR $0.84 ; 95 \%$ CI $0.73,0.95$ for diabetes and RR $0.91,95 \%$ CI $0.84,0.98$ for prediabetes per additional year of menarche).

Fasting glucose levels were $-0.008 \mathrm{mmol} / \mathrm{l}(95 \% \mathrm{CI}-0.012$, -0.003 ) per additional year of age at menarche, adjusted for year of birth. After multivariable adjustment, FG levels were $-0.007(95 \%$ CI $-0.011,-0.002)$ per additional year of age at menarche. After further adjustment of current BMI, the fasting blood glucose levels were $-0.004(95 \%$ CI $-0.008,0.001)$ per additional year of age at menarche. The corresponding $B$-estimates and $95 \% \mathrm{CI}$ for the association between age at menarche and

Table 1 Selected characteristics by NGT, i-IFG, i-IGT, IFG-IGT, NDD and known diabetes $(N=1,503)$

\begin{tabular}{|c|c|c|c|c|c|c|}
\hline Variable & NGT & i-IFG & i-IGT & IFG-IGT & NDD & Known diabetes \\
\hline$n$ & 1,137 & 36 & 163 & 27 & 45 & 95 \\
\hline Age (years) & $52.8 \pm 12.4$ & $61.7 \pm 11.0$ & $63.5 \pm 11.4$ & $66.1 \pm 7.1$ & $64.9 \pm 10.6$ & $66.2 \pm 9.4$ \\
\hline Age at menarche (years) & $13.5 \pm 1.5$ & $13.3 \pm 1.8$ & $13.6 \pm 1.6$ & $13.3 \pm 1.4$ & $13.5 \pm 2.0$ & $13.4 \pm 1.8$ \\
\hline BMI $\left(\mathrm{kg} / \mathrm{m}^{2}\right)$ & $26.2 \pm 4.8$ & $29.8 \pm 6.6$ & $29.4 \pm 4.9$ & $31.3 \pm 4.9$ & $31.5 \pm 4.1$ & $33.1 \pm 6.0$ \\
\hline $\mathrm{WC}(\mathrm{cm})$ & $85.1 \pm 11.9$ & $96.4 \pm 13.7$ & $93.2 \pm 12.2$ & $101.6 \pm 13.0$ & $100.1 \pm 9.3$ & $102.5 \pm 13.6$ \\
\hline BMI at age 25 years $\left(\mathrm{kg} / \mathrm{m}^{2}\right)$ & $22.1 \pm 3.3$ & $22.6 \pm 4.13$ & $22.8 \pm 2.9$ & $22.8 \pm 3.0$ & $23.1 \pm 3.2$ & $24.2 \pm 5.1$ \\
\hline $\begin{array}{l}\mathrm{HbA}_{1 \mathrm{c}}(\%, \text { proportion } \\
\text { of total haemoglobin })\end{array}$ & $5.4 \pm 0.3$ & $5.7 \pm 0.3$ & $5.6 \pm 0.3$ & $6.0 \pm 0.4$ & $6.2 \pm 0.6$ & $6.8 \pm 1.0$ \\
\hline $\mathrm{HbA}_{1 \mathrm{c}}(\mathrm{mmol} / \mathrm{l})$ & $35.5 \pm 1.5$ & $38.8 \pm 1.5$ & $37.7 \pm 1.5$ & $42 \pm 2.0$ & $44.3 \pm 3.0$ & $50.8 \pm 5.0$ \\
\hline $\mathrm{FG}(\mathrm{mmol} / \mathrm{l})^{\mathrm{a}}$ & $4.9(4.67,5.22)$ & $6.2(6.14,6.33)$ & $5.3(4.94,5.67)$ & $6.6(6.39,6.72)$ & $6.6(5.94,7.17)$ & - \\
\hline 2-h glucose $(\mathrm{mmol} / \mathrm{l})^{\mathrm{a}}$ & $5.4(4.56,6.28)$ & $6.5(5.42,6.97)$ & $8.7(8.17,9.56)$ & $8.9(8.44,9.61)$ & $12.4(11.33,13.67)$ & - \\
\hline $\mathrm{TG}(\mathrm{mmol} / \mathrm{l})^{\mathrm{a}}$ & $1.0(0.70,1.35)$ & $1.4(1.15,2.04)$ & $1.3(0.95,1.62)$ & $1.9(1.41,2.39)$ & $1.7(1.23,2.62)$ & - \\
\hline Education $(\leq 10$ years $)$ & 43.6 & 47.2 & 63.8 & 59.3 & 71.1 & 73.7 \\
\hline $\begin{array}{l}\text { Married or living } \\
\text { with a partner (\%) }\end{array}$ & 70.8 & 66.7 & 67.5 & 55.6 & 62.2 & 62.1 \\
\hline Postmenopausal (\%) & 42.2 & 72.2 & 73.0 & 88.9 & 77.8 & 78.7 \\
\hline Parity $(\geq 3)(\%)$ & 34.4 & 41.7 & 38.7 & 37.0 & 51.1 & 45.3 \\
\hline Alcohol $>20$ g/day $(\%)$ & 15.6 & 25.0 & 11.0 & 14.8 & 15.6 & 8.4 \\
\hline Current smoker (\%) & 17.7 & 11.1 & 8.0 & 11.1 & 8.9 & 9.5 \\
\hline Physically active during leisure time (\%) & 58.7 & 33.3 & 52.8 & 59.3 & 42.2 & 38.9 \\
\hline Actual hypertension $(\%)^{\mathrm{b}}$ & 24.4 & 52.8 & 44.8 & 55.6 & 80.0 & 81.1 \\
\hline
\end{tabular}

Data are expressed as mean $\pm \mathrm{SD}$, median (25th to 75 th percentiles) or percentages

${ }^{\mathrm{a}}$ These variables are measured in all fasting individuals, therefore $n=1,408 ; 2$-h glucose was measured after administration of an OGTT in all nondiabetic participants

${ }^{\mathrm{b}}$ Defined as use of antihypertensive medication, being aware of having hypertension or blood pressure values greater than $140 / 90 \mathrm{mmHg}$ 
Table 2 Selected characteristics by age at menarche (categorised)

Data are expressed as mean $\pm \mathrm{SD}$, median (25th to 75 th percentile) or percentages

${ }^{a}$ Due to a non-normal distribution these variables were $\log$ transformed for calculation of the $p$ value and the results are presented as median (25th to 75th percentile)

${ }^{\mathrm{b}}$ These variables are measured in all fasting individuals therefore $n=1,408$; 2-h glucose was measured after administration of an OGTT in all non-diabetic participants

${ }^{\mathrm{c}}$ Defined as use of antihypertensive medication, being aware of having hypertension or blood pressure values greater than 140/90 $\mathrm{mmHg}$

${ }^{\mathrm{d}} p$ value, testing the difference between the three categories of age at menarche

\begin{tabular}{|c|c|c|c|c|}
\hline \multirow[t]{2}{*}{ Characteristic } & \multicolumn{3}{|c|}{ Age at menarche (years) } & \multirow[t]{2}{*}{$p$ value } \\
\hline & $<12$ & $12-15$ & $>15$ & \\
\hline$n$ & 117 & 1,234 & 152 & \\
\hline Age (years) & $53.2 \pm 12.1$ & $55.1 \pm 12.9$ & $61.6 \pm 13.2$ & $<0.001$ \\
\hline BMI $\left(\mathrm{kg} / \mathrm{m}^{2}\right)$ & $28.1 \pm 5.7$ & $27.3 \pm 5.3$ & $27.1 \pm 5.0$ & 0.202 \\
\hline $\mathrm{WC}(\mathrm{cm})$ & $89.6 \pm 14.0$ & $88.0 \pm 13.3$ & $87.9 \pm 12.7$ & 0.455 \\
\hline BMI at age 25 years $\left(\mathrm{kg} / \mathrm{m}^{2}\right)$ & $23.4 \pm 4.4$ & $22.3 \pm 3.3$ & $22.5 \pm 3.4$ & 0.003 \\
\hline $\begin{array}{l}\mathrm{HbA}_{1 \mathrm{c}}(\%, \text { proportion of total } \\
\text { haemoglobin) }\end{array}$ & $5.5 \pm 0.5$ & $5.5 \pm 0.5$ & $5.6 \pm 0.6$ & 0.117 \\
\hline $\mathrm{HbA}_{1 \mathrm{c}}(\mathrm{mmol} / \mathrm{mol})$ & $36.6 \pm 1.5$ & $36.6 \pm 1.5$ & $37.7 \pm 3.0$ & \\
\hline $\mathrm{FG}(\mathrm{mmol} / \mathrm{l})^{\mathrm{a}} \mathrm{b}$ & $5.32(4.78,5.61)$ & $5.18(4.72,5.44)$ & $5.32(4.78,5.53)$ & 0.404 \\
\hline 2-h glucose $(\mathrm{mmol} / \mathrm{l})^{\mathrm{a}} \mathrm{b}$ & $6.01(4.67,7.11)$ & $5.80(4.78,6.94)$ & $5.98(5.06,7.28)$ & 0.334 \\
\hline $\mathrm{TG}(\mathrm{mmol} / \mathrm{l})^{\mathrm{a} b}$ & $1.2(0.80,1.62)$ & $1.0(0.73,1.45)$ & $1.1(0.86,1.59)$ & 0.121 \\
\hline Education $(\leq 10$ years $)(\%)$ & 58.7 & 48.0 & 56.6 & 0.137 \\
\hline Married or living with a partner $(\%)$ & 70.9 & 70.0 & 63.2 & 0.058 \\
\hline Postmenopausal (\%) & 43.6 & 48.9 & 68.4 & $<0.001$ \\
\hline Parity ( $\geq 3)(\%)$ & 35.0 & 35.9 & 40.1 & 0.327 \\
\hline Alcohol >20 g/day (\%) & 17.9 & 15.0 & 10.5 & 0.383 \\
\hline Current smoker $(\%)$ & 19.7 & 15.5 & 13.2 & 0.339 \\
\hline $\begin{array}{l}\text { Physically active during leisure } \\
\text { time }(\%)\end{array}$ & 41.9 & 56.7 & 59.2 & 0.006 \\
\hline Actual hypertension ${ }^{\mathrm{c}}(\%)$ & 33.2 & 32.3 & 39.5 & 0.205 \\
\hline
\end{tabular}

2-h glucose were -0.015 (95\% CI $-0.024,-0.005),-0.015$ (95\% CI $-0.024,-0.005)$ and -0.009 (95\% CI -0.019 , $-0.0001)$ per additional year of age at menarche. Similar results were obtained for the model that adjusted for BMI at age 25 years instead of current BMI for both glucose values (data not shown).

\section{Discussion}

This study showed that earlier age at menarche is significantly associated with prediabetes and diabetes, even after adjustment for relevant confounders and current BMI. Therefore, our study provides further evidence that low age at menarche is an independent risk factor for diabetes and does not only impact type 2 diabetes risk through its effects on BMI.

Age at menarche is an indicator of maturation and timing of puberty in girls. Age at menarche has been fallen from 17 years on average since the nineteenth century, to 13 years on average in many European countries, a level at which it has stayed relatively stable since the 1960s [25]. This effect may be due to changes in the socioeconomic and demographic environment, possibly acting through changes in nutrition and exercise patterns [26]. There is evidence that younger age at menarche might lead to certain chronic diseases $[8-10,12]$. Whether this secular trend in puberty timing is really associated with adverse health implications still needs to be assessed [27].

Knowing that forms of prediabetes, like IFG and IGT, carry an increased risk of type 2 diabetes [20], this study not only investigated associations for age at menarche with diabetes, but also examined prediabetes to improve knowledge about populations at risk. The Rancho Bernardo Study, to our knowledge the only study besides this work to examine abnormal glucose tolerance by administration of an OGTT, found no association between age at menarche and abnormal glucose tolerance and type 2 diabetes in 997 women aged 50-92 years [7]. Although this might represent the absence of such an association, it might also simply be the result of insufficient power or due to the fact that only postmenopausal women were examined. The authors found that late age at menarche was inversely associated with fasting and post-challenge glycaemia. Our results confirm these findings.

The few other studies on the association between age at menarche and previously diagnosed type 2 diabetes [5-7, 14] show inconclusive results. Our findings are comparable with those of the Nurses' Health Study I (101,415 women, age range 34-59 years) and II (100,547 women, age range 26-46 years) [6], and the prospective EPIC-Norfolk cohort study (13,308 women, age range 40-75 years) [5], which showed that age at menarche is inversely associated with diabetes. The EPIC-Norfolk study suggested that age at 
Table 3 Association of age at menarche with prediabetes and diabetes (Poisson analysis)

\begin{tabular}{llll}
\hline Value & $\begin{array}{l}\text { Prediabetes or } \\
\text { diabetes }(n=366)\end{array}$ & $\begin{array}{l}\text { Combined } \\
\text { prediabetes } \\
(n=226)\end{array}$ & $\begin{array}{l}\text { Diabetes } \\
(n=140)\end{array}$ \\
\hline Birth year-adjusted RR & 0.88 & 0.91 & 0.83 \\
95\% CI & $0.82,0.94$ & $0.84,0.98$ & $0.73,0.95$ \\
$p$ value & $<0.0001$ & 0.015 & 0.007 \\
Multivariable-adjusted & 0.88 & 0.91 & 0.83 \\
$\quad$ RR & $0.83,0.94$ & $0.85,0.98$ & $0.73,0.95$ \\
$95 \%$ CI & 0.0001 & 0.019 & 0.007 \\
$p$ value & 0.89 & 0.92 & 0.84 \\
Multivariable+current & & & \\
$\quad$ BMI-adjusted RR & $0.83,0.95$ & $0.85,0.99$ & $0.73,0.98$ \\
95\% CI & 0.0009 & 0.036 & 0.016 \\
$p$ value & & & \\
\hline
\end{tabular}

$N=1,503, n(\mathrm{NGT})=1,137$

Prediabetes or diabetes: all cases (prediabetes and diabetes) combined in one group as outcome

Combined prediabetes: i-IFG, i-IGT and IFG-IGT combined in one group, for this analysis the diabetes cases were excluded, resulting in $n=1,363$ for the analyses

Diabetes: newly diagnosed and previously diagnosed diabetes combined in one group, for this analysis the prediabetic women (i-IFG, iIGT and IFG-IGT) were considered as NGT

Multivariable-adjustment: adjusted for year of birth, physical activity, education, marital status, smoking, alcohol consumption and menopausal status

Model 2+3: $n=1,498$ due to five missing values of date of diabetes diagnosis

NGT was used as a reference. The reported associations are per additional year of age at menarche

menarche and its association with diabetes is completely mediated by adult obesity [5]. The Nurses' Health Study showed an increased risk of type 2 diabetes in women with early menarche, with a stronger effect in younger than older women, and this effect seems to be mediated through excessive adult adiposity. The association among younger and middle-aged women (Nurses' Health Study II) could not be fully explained by increased adult BMI, suggesting a risk pathway between age at menarche and type 2 diabetes beyond excessive adiposity [6]. Our study provides support for this finding, as the RR for diabetes only marginally changed after adjustment for various confounders, including current BMI or BMI at age 25 years. Two smaller studies found no association between age at menarche and type 2 diabetes, even without adjustment for BMI: the prospective Menstruation and Reproductive History Study of 668 women with an age range of 63-81 [14], and the cross-sectional Rancho Bernardo Study in 997 women aged 50-92 [7].

Childhood anthropometric measurements and obesity play an important role in understanding adult obesity and type 2 diabetes as well as the onset of puberty. Prior studies have reported that the inverse association of age at menarche with BMI and obesity in middle age is not explained by early childhood BMI confounding [18, 19]. There is evidence that childhood socioeconomic status (SES) is associated with type 2 diabetes and obesity in later adulthood [28], and other results suggest that lower SES at 7 years and reductions in SES in early childhood are both associated with an earlier age at menarche [29]. In this study we could not adjust for SES or obesity in childhood, a question that future studies should address.

Genetic factors also play an important role in the variation in the timing of puberty [15]. It is estimated that approximately half the phenotypic variation among girls from high-income countries in the timing of puberty is due to genetic factors $[30,31]$. An association has been found between decreased birthweight and diabetes type 2 risk alleles [32]. Another study showed an association between higher birthweight with later onset of age at menarche [33]. Diabetes is caused by multigenetic factors and environmental factors as well, so we suggest there might be a link. In addition, early menarche seems to be a measure for hormonal imbalance [34], which has been shown to be associated with diabetes [35]. Therefore, different pathways beyond mediating BMI might be possible. Our study supports that the association of age at menarche with diabetes is not merely mediated by BMI. Menarche can be delayed by early metformin therapy in low-birthweight girls with precocious pubarche [30], but whether this reduces the risk of chronic diseases in later life needs to be explored.

Strengths and limitations One strength of the current study is its representativeness, as results are based on a large sample, drawn from the general population. Moreover, the accurate measurement of prediabetes and diabetes by OGTT or previously known diabetes confirmed by physicians is an advantage compared with other studies that rely on self-report. Another strong point is the availability of data on lifestyle factors measured according to a standardised protocol. Compared with other studies, weight and height data are based on measurements rather than self-reported data, which reduces biases as participants tend to under-report weight and overreport height [31]. Furthermore, this study is based on a wider age range (between 32 and 81 years) than other studies, which only show results for postmenopausal female participants or for participants in young adulthood.

Several limitations need to be stated. The variable age at menarche has been assessed retrospectively, therefore misclassification may have occurred. Nevertheless, it has been shown that the actual reported mean age at menarche is not that different from the mean age at menarche recalled 33 years later $[36,37]$. In addition, misclassification of age at menarche is not likely to be associated with diabetes. Misclassification of confounding variables might weaken the observed 
associations by regression dilution as some of the covariables were measured by self-report, such as alcohol consumption, smoking habits and physical activity. Because of the observational nature of our publication we cannot, as in all observational studies, rule out the possibility of residual confounding due to unmeasured or poorly measured confounders. No information was available on history of gestational diabetes. Lack of power is another restriction. A further limitation of this study is its cross-sectional design, implicating that cause and effect relationships cannot be discerned.

Conclusion and implication The present study showed that an earlier age at menarche plays a role in the development of prediabetes and diabetes independently of BMI. Thus a history of early menarche may help to identify women at risk for diabetes. Early detection of women at risk may help to prevent diabetes and its subsequent morbidity. Further research should focus on underlying genetic links with age at menarche and diabetes, and longitudinal studies are essential to assess the risk of diabetes due to childhood factors, reproductive variables and risk of diabetes.

Acknowledgements We thank all the study participants, all members of staff at the Institute of Epidemiology II and the field staff in Augsburg who planned and conducted the study.

Funding The KORA research platform (KORA, Cooperative Research in the Region of Augsburg) was initiated and financed by the Helmholtz Zentrum München, German Research Center for Environmental Health $(\mathrm{GmbH})$, which is funded by the German Federal Ministry of Education, Science, Research and Technology and by the State of Bavaria. C. Huth was supported by a grant from the German Federal Ministry of Education and Research (BMBF) to the German Center for Diabetes Research (DZD e.V.).

Duality of interest The authors declare that there is no duality of interest associated with this manuscript.

Contribution statement DS planned the study, contributed to the data acquisition, performed the data analysis and wrote the manuscript. $\mathrm{AD}$ and $\mathrm{CM}$ contributed to the acquisition of data, planning of the study, the performance of the data analysis and its interpretation and reviewed and edited the manuscript extensively. $\mathrm{CH}, \mathrm{BT}, \mathrm{AP}$ and $\mathrm{MH}$ contributed to the acquisition of data, data analysis and interpretation and reviewed and edited the manuscript. HS, WR and BK contributed to the data analysis and data interpretation and reviewed and edited the manuscript. All authors have viewed and approved the final version of the manuscript.

\section{References}

1. Mokdad AH, Ford ES, Bowman BA et al (2003) Prevalence of obesity, diabetes, and obesity-related health risk factors, 2001. JAMA 289:76-79
2. Rathmann W, Haastert B, Icks A et al (2003) High prevalence of undiagnosed diabetes mellitus in Southern Germany: target populations for efficient screening. The KORA survey 2000. Diabetologia 46:182-189

3. Meisinger C, Strassburger K, Heier M et al (2010) Prevalence of undiagnosed diabetes and impaired glucose regulation in 35-59year-old individuals in Southern Germany: the KORA F4 Study. Diabet Med 27:360-362

4. Chen L, Zhang C, Yeung E et al (2011) Age at menarche and metabolic markers for type 2 diabetes in premenopausal women: the BioCycle Study. J Clin Endocrinol Metab 96:E1007-E1012

5. Lakshman R, Forouhi N, Luben R et al (2008) Association between age at menarche and risk of diabetes in adults: results from the EPIC-Norfolk cohort study. Diabetologia 51:781-786

6. He C, Zhang C, Hunter DJ et al (2010) Age at menarche and risk of type 2 diabetes: results from 2 large prospective cohort studies. Am J Epidemiol 171:334-344

7. Saquib N, Kritz-Silverstein D, Barrett-Connor E (2005) Age at menarche, abnormal glucose tolerance and type 2 diabetes mellitus: the Rancho Bernardo Study. Climacteric 8:76-82

8. Kivimaki M, Lawlor DA, Smith GD et al (2008) Association of age at menarche with cardiovascular risk factors, vascular structure, and function in adulthood: the Cardiovascular Risk in Young Finns Study. Am J Clin Nutr 87:1876-1882

9. Lakshman R, Forouhi NG, Sharp SJ et al (2009) Early age at menarche associated with cardiovascular disease and mortality. J Clin Endocrinol Metab 94:4953-4960

10. Remsberg KE, Demerath EW, Schubert CM, Chumlea WC, Sun SS, Siervogel RM (2005) Early menarche and the development of cardiovascular disease risk factors in adolescent girls: the Fels Longitudinal Study. J Clin Endocrinol Metab 90:2718-2724

11. Jacobsen BK, Heuch I, Kvale G (2007) Association of low age at menarche with increased all-cause mortality: a 37-year follow-up of 61,319 Norwegian women. Am J Epidemiol 166:1431-1437

12. Jacobsen BK, Oda K, Knutsen SF, Fraser GE (2009) Age at menarche, total mortality and mortality from ischaemic heart disease and stroke: the Adventist Health Study, 1976-88. Int J Epidemiol 38:245-252

13. Stöckl D, Meisinger C, Peters A et al (2011) Age at menarche and its association with the metabolic syndrome and its components: results from the KORA F4 Study. PLoS One 6:e26076

14. Cooper GS, Ephross SA, Sandler DP (2000) Menstrual patterns and risk of adult-onset diabetes mellitus. J Clin Epidemiol 53:1170-1173

15. Elks CE, Perry JR, Sulem P et al (2010) Thirty new loci for age at menarche identified by a meta-analysis of genome-wide association studies. Nat Genet 42:1077-1085

16. Unwin N, Shaw J, Zimmet P, Alberti KG (2002) Impaired glucose tolerance and impaired fasting glycaemia: the current status on definition and intervention. Diabet Med 19:708-723

17. van Lenthe FJ, Kemper CG, van Mechelen W (1996) Rapid maturation in adolescence results in greater obesity in adulthood: the Amsterdam Growth and Health Study. Am J Clin Nutr 64:1824

18. Pierce MB, Leon DA (2005) Age at menarche and adult BMI in the Aberdeen Children of the 1950s Cohort Study. Am J Clin Nutr 82:733-739

19. Parsons TJ, Power C, Logan S, Summerbell CD (1999) Childhood predictors of adult obesity: a systematic review. Int J Obes Relat Metab Disord 23(Suppl 8):S1-S107

20. Rathmann W, Strassburger K, Heier M et al (2009) Incidence of type 2 diabetes in the elderly German population and the effect of clinical and lifestyle risk factors: KORA S4/F4 cohort study. Diabet Med 26:1212-1219

21. Meisinger C, Thorand B, Schneider A, Stieber J, Doring A, Lowel $\mathrm{H}$ (2002) Sex differences in risk factors for incident type 2 diabetes 
mellitus: the MONICA Augsburg cohort study. Arch Intern Med $162: 82-89$

22. WHO (1999) Definition, diagnosis and classification of diabetes mellitus and its complications. In: Part 1: Diagnosis and classification of diabetes mellitus. Report of a WHO consultation. WHO, Geneva

23. Forouhi NG, Balkau B, Borch-Johnsen K et al (2006) The threshold for diagnosing impaired fasting glucose: a position statement by the European Diabetes Epidemiology Group. Diabetologia 49:822-827

24. Zou G (2004) A modified Poisson regression approach to prospective studies with binary data. Am J Epidemiol 159:702-706

25. Gohlke B, Woelfle J (2009) Growth and puberty in German children: is there still a positive secular trend? Deutsches Arzteblatt International 106:377-382

26. Okasha M, McCarron P, McEwen J, Smith GD (2001) Age at menarche: secular trends and association with adult anthropometric measures. Ann Hum Biol 28:68-78

27. Golub MS, Collman GW, Foster PM et al (2008) Public health implications of altered puberty timing. Pediatrics 121(Suppl 3): S218-S230

28. Tamayo T, Christian H, Rathmann W (2010) Impact of early psychosocial factors (childhood socioeconomic factors and adversities) on future risk of type 2 diabetes, metabolic disturbances and obesity: a systematic review. BMC Public Health 10:525

29. James-Todd T, Tehranifar P, Rich-Edwards J, Titievsky L, Terry MB (2010) The impact of socioeconomic status across early life on age at menarche among a racially diverse population of girls. Ann Epidemiol 20:836-842

30. Ibanez L, Lopez-Bermejo A, Diaz M, Marcos MV, de Zegher F (2011) Early metformin therapy to delay menarche and augment height in girls with precocious pubarche. Fertil Steril 95:727-730

31. Rowland ML (1990) Self-reported weight and height. Am J Clin Nutr 52:1125-1133

32. Andersson EA, Pilgaard K, Pisinger C et al (2010) Type 2 diabetes risk alleles near ADCY5, CDKAL1 and HHEX-IDE are associated with reduced birthweight. Diabetologia 53:1908-1916

33. Ruder EH, Hartman TJ, Rovine MJ, Dorgan JF (2010) Birth characteristics and age at menarche: results from the Dietary Intervention Study in Children (DISC). Cancer Causes Control 21:1379-1386

34. Vihko R, Apter D (1984) Endocrine characteristics of adolescent menstrual cycles: impact of early menarche. J Steroid Biochem 20:231-236

35. Ding EL, Song Y, Malik VS, Liu S (2006) Sex differences of endogenous sex hormones and risk of type 2 diabetes: a systematic review and meta-analysis. JAMA 295:1288-1299

36. Must A, Phillips SM, Naumova EN et al (2002) Recall of early menstrual history and menarcheal body size: after 30 years, how well do women remember? Am J Epidemiol 155:672-679

37. Bosetti C, Tavani A, Negri E, Trichopoulos D, La Vecchia C (2001) Reliability of data on medical conditions, menstrual and reproductive history provided by hospital controls. J Clin Epidemiol 54:902-906 